\title{
Mujeres en la universidad: presencias y experiencias biográficas San Luis Potosí, México 1950-1970
}

\author{
Blanca Susana Vega Martínez ${ }^{2}$ \\ Universidad Autónoma de San Luis Potosí, México
}

Recepción: 30/09/2020

Evaluación: 02/10/2020

Aceptación: 15/10/2020

Artículo de Investigación-Científica

DOI: https://doi.org/10.22267/rhec.202525.81

\section{Resumen}

Este artículo surge de una investigación más amplia, que tiene como objetivo recuperar las trayectorias educativas de mujeres universitarias que ejercieron su profesión entre las décadas de 1950 y 1970; asimismo, reconocer las experiencias que tuvieron al acceder a la educación superior en la Universidad Autónoma de San Luis Potosí (UASLP), México. El acceso a la educación, para las mujeres, ha sido un proceso discontinuo en relación con el de los hombres, por lo que su participación en el ámbito educativo y académico se ha sobrellevado con dificultades y buscando caminos diversos. Actualmente jubiladas y en proceso de envejecimiento, las universitarias nos narran su trayectoria educativa en un tiempo de difícil acceso a la educación superior femenina, sobre todo en carreras consideradas tradicionalmente de enseñanza masculina, es decir, medicina, ciencias químicas, enfermería y derecho. La metodología se enfoca en el método biográfico, específicamente en los relatos de vida, que nos permiten recuperar, desde la propia voz de las universitarias, las experiencias en su

1 Este artículo se deriva de la investigación: "La participación de las mujeres en la Educación Superior en San Luis Potosí" avalada por la Universidad Autónoma de San Luis Potosí, México

2 Docente investigadora de la Universidad Autónoma de San Luis Potosí, México. Grupo de investigación: Psicología y Educación-UASLP; Eleko: histórias, culturas e experiências formativas Universidade do Estado do Rio de Janeiro-UERJ; História e Estudos de Gênero, Universidade Federal de Mato Grosso, Brasil. Línea de investigación: Historia de las mujeres; Educación, género y sociedad. Correo electrónico: susana.vega@uaslp. mx. (i) https://orcid.org/0000-0002-5298-9442 
trayectoria educativa y laboral en las décadas de 1950 a 1970, primeramente, por ser mujeres estudiantes universitarias y, posteriormente, al ejercer su profesión en los ámbitos universitarios y fuera de ellos.

Palabras clave: Educación de la mujer; Educación superior; México.

\title{
Women in higher education: presence and autobiographic experiences San Luis Potosí, México 1950-1970
}

\begin{abstract}
This article is a product of a deeper academic research, which aimed to recover the educational trajectories of women in higher education who practiced their profession between the 1950s and 1970s; also, to recognize the experiences they had when accessing higher education at the Universidad Autónoma de San Luis Potosí (UASLP) in Mexico. For women, access to education has been a discontinuous process when compared to men, so that their participation in the educational and academic field has been overcome with difficulties and seeking different paths. Currently retired and in the process of aging, women in higher education tell the story about their educational trajectory in a time of difficult access to higher education for women, especially in traditional fields considered to be for men, for example, medicine, chemical sciences, nursing and law. The methodology focuses on the biographical method, specifically on life stories, therefore, it was possible to retrieve the experiences in women's educational and labor trajectory in the decades from 1950 to 1970 , firstly, as students in higher educations and, later, when practicing their profession in and out of the university.
\end{abstract}

Keywords: Women education; higher education; Mexico. 


\section{Mulheres na universidade: presenças e experiências biográficas San Luis Potosí, México 1950-1970}

\section{Resumo}

Este artigo surge de uma investigação mais ampla, que visa resgatar as trajetórias educacionais de mulheres universitárias que exerceram sua profissão entre as décadas de 1950 e 1970; também, reconhecer as experiências que tiveram no acesso ao ensino superior na Universidade Autônoma de San Luis Potosí (UASLP), México. O acesso à educação, para as mulheres, tem sido um processo descontínuo em relação aos homens, por isso sua participação na esfera educacional e acadêmica tem sido superada com dificuldades e buscando diferentes caminhos. Atualmente aposentadas e em processo de envelhecimento, as universitárias nos contam sobre sua trajetória educacional em um momento de difícil acesso ao ensino superior feminino, principalmente em carreiras tradicionalmente consideradas como educação masculina, ou seja, medicina, ciências químicas, enfermagem e direito. A metodologia centra-se no método biográfico, especificamente nas histórias de vida, que nos permitem recuperar, a partir da voz das universitárias, as experiências da sua trajetória educacional e de trabalho nas décadas de 1950 a 1970 , em primeiro lugar, por serem mulheres. estudantes universitárias e, posteriormente, no exercício da profissão em ambiente universitário e fora dele.

Palavras-chave: Educação da mulher; Educação superior; México.

\section{Introducción}

La Historia de mujeres es un campo de la historiografía que reconoce y conceptualiza a las mujeres como sujetas a la Historia y a la construcción de acontecimientos, experiencias y creación de cambios sociales. Busca reconocer las aportaciones y situaciones que las mujeres han vivido a lo largo de la Historia. Como campo de estudio, además, demanda romper 
con el relato universal que solo da voz a la narrativa masculina, y que ha generado una producción de conocimiento que hace de las mujeres un constante cuestionamiento.

Por ello, en este texto, se tejen dos campos de estudio, la Historia de las mujeres, específicamente su educación, y las experiencias biográficas, con la intención de explicar las condiciones que vivieron las mujeres que se incorporaron a la educación superior universitaria durante las décadas de 1950 a 1970 en la ciudad de San Luis Potosí, México.

De la voz de cuatro mujeres universitarias mostramos tres dimensiones de análisis: el acceso a la educación superior universitaria, las condiciones que les permitieron su ingreso, y las relaciones de género que gestaron en su cotidianidad escolar y/o social; especialmente, damos cuenta de las jerarquías impuestas en las relaciones de género que se interponen en los espacios escolares y laborales de las profesoras. Por ello, consideramos indispensable conocer el origen histórico y político de la jerarquización, inequidad, y desigualdad que se produjo en las relaciones de género de nuestras entrevistadas, a la vez que mostramos cómo sortearon esas relaciones, y cómo incidieron en su posicionamiento y agencia.

Este texto forma parte de una investigación, que aún sigue en curso, y cuyo objetivo es indagar las condiciones sociales de las estudiantes y profesoras universitarias que incursionaron en la Universidad Autónoma de San Luis Potosí entre las décadas de 1950 y 1970, a través de entrevistas biográficas. La investigación continúa en la búsqueda de nuevos relatos, experiencias y conocimiento del acceso a la educación superior de las mujeres en el Estado; con ello, privilegiamos los ámbitos locales y procesos micro sociales.

En un primer momento, mostramos una breve contextualización de la educación de las mujeres en México hacia la segunda mitad del siglo XX, específicamente en las décadas de 1960 a 1980.

Posteriormente, mostramos el vínculo de las experiencias biográficas y la memoria, para reconocer cómo la memoria reúne dos aspectos fundamentales: la posición ética de quien escucha y la posición política de quien narra.

Por último, en un tercer apartado se presentan las narrativas de las cuatro mujeres; cada una de ellas nos revela su posicionamiento en los espacios universitarios y laborales, que se presentan a través de sus experiencias biográficas; es decir, se partió de la posibilidad de utilizar el método 
biográfico como forma de acercamiento a las mujeres que accedieron a la educación superior universitaria.

El acercamiento con estas mujeres se dio de manera paulatina; se realizaron varias entrevistas, en distintos espacios físicos, tales como: las casas propias de las participantes, la misma universidad e incluso cafeterías y restaurantes. En las entrevistas, las mujeres nos relataron sus experiencias escolares a lo largo de su trayectoria educativa; especialmente, su ingreso a la educación superior, sus experiencias laborales tanto dentro de la universidad como fuera de ella, así como asuntos familiares, redes de apoyo y su incursión en espacios considerados socialmente masculinos. Posteriormente, se transcribieron las entrevistas y se llevó a cabo su sistematización para la construcción de relatos de vida.

Cabe resaltar que, si bien los relatos de vida se ubican en el espacio escolar y profesional de las mujeres, específicamente en reconocer cuáles fueron las condiciones que les permitieron acceder a los espacios universitarios, qué limitaciones sortearon y qué posibilidades y/o privilegios tuvieron, no podemos olvidar que "la vida humana, la vivencia humana no puede cortarse y ponerse en compartimentos de espacios y temporalidades autónomas e impermeables los unos con respecto a los otros"; 3 es decir, a pesar de que se plasmase en este texto solo una parte o fragmento de los relatos de vida de estas mujeres, no podemos negar que los conforman otros aspectos sociales y colectividades a los cuales pertenecieron.

Al seguir a Delory-Momberger, nos señala:

-sin importar las fragmentaciones, las rupturas, algunas veces los impases de la narración- [los relatos] expresan el trabajo de interpretación y de construcción que los actores cumplen sobre ellos mismos, sobre su existencia, sobre sus relaciones con los demás, sobre su lugar en sus diversos ambientes sociales. ${ }^{4}$

Ahora bien, las participantes de este trabajo fueron cuatro mujeres que oscilaban entre los 62 y los 85 años de edad en el momento de sus entrevistas, y las cuales accedieron a la educación superior entre las décadas de 1950 y 1970; actualmente, tres son jubiladas y en proceso de

3 Christine Delory-Momberger, La condición biográfica (Medellín: Universidad de Antioquia, 2015), 45.

4 Delory-Momberger, La condición biográfica, 45. 
envejecimiento, mientras que una continúa en el ejercicio de su profesión. Las entrevistadas pertenecieron a tres escuelas: la Escuela de Medicina (con las carreras de enfermería y medicina), la Escuela de Ciencias Químicas, y la Escuela de Derecho. Las profesoras de mayor edad pertenecieron a las dos primeras escuelas, mientras que la profesora de menor edad perteneció a la Escuela de Derecho. Aún no se tienen datos precisos en la universidad sobre las primeras estudiantes en cursar y presentar examen profesional en la carrera de enfermería, pero data de la década de 1920; por ello, la carrera de enfermería y obstetricia tiene un reconocimiento en la incorporación de las mujeres como estudiantes desde sus inicios, mas no como profesoras -las cuales se incorporan hasta la década de 1960-; mientras la carrera de medicina tuvo la presencia de contadas estudiantes entrado el siglo XX y, como profesoras, hasta la década de 1930. Por su parte, la Escuela de Ciencias Químicas se caracterizó por ser una Escuela con gran aceptación de las mujeres entre el estudiantado desde principios del siglo XX, más como profesoras hasta 1945. Cabe señalar incluso que a la carrera de Ingeniería química industrial se la consideraba propia de los varones, y una de nuestras entrevistadas perteneció a esa carrera; por último, la Escuela de Derecho se la ha considerado propiamente masculina, con mínima aceptación de estudiantes y profesoras entre sus aulas, a quienes se incorporó después de 1950. Por ello, la profesora de menor edad fue una de las primeras profesoras incorporadas como parte de la planta docente de esta Escuela hacia finales de la década de $1970 .^{5}$

En las entrevistas, nos narran sus experiencias al ser de las primeras generaciones en pertenecer al gremio estudiantil, así como al profesorado en su Escuela de formación. Como parte de la pertinencia ética de la investigación, se recurrió al consentimiento informado, en el que se les dieron a conocer los pormenores de la investigación, así como los acuerdos para la presentación de sus experiencias.

Por último, es importante reconocer que la experiencia biográfica nos permite retomar de viva voz cómo las mujeres habitaron espacios, se

\footnotetext{
5 Para mayor información sobre la incorporación de estudiantes y profesoras en la universidad, véase: Blanca Susana Vega, "La participación de las estudiantes y profesoras en las aulas universitarias: San Luis Potosí, 1923-1952" en: Los mundos escolares en el noreste de México: ruralidad y género en el siglo XX, comps. Mónica Chávez González y René Medina (UNAM-BECENE, 2019), 113-136; Rosa María Huerta, "Ingreso y presencia de las mujeres en la matrícula universitaria en México". Revista de El Colegio de San Luis, [s.1.], No 14, (2017): 282-306.
} 
construyeron y configuraron sus identidades, se posicionaron y permitieron a través de su cohabitar impulsar a otras mujeres que les sucedieron.

\section{La educación de las mujeres en México (1950-1970)}

Tuñón ${ }^{6}$ nos señala que la declaración de la educación primaria como obligatoria en 1878 para ambos sexos representó una medida de índole liberal y un cambio gradual. Unos años después, hacia 1890, con la apertura de la Escuela Normal para Señoritas, el magisterio se convirtió en una de las primeras opciones de profesionalización de las mujeres en México. Ello representó una conquista de los espacios de educación superior en México por las mujeres. Sin embargo, mientras que la mujer tuvo una mayor apertura en las Escuelas normalistas, el acceso de las mujeres a la universidad tuvo muy poca presencia; el registro de las primeras mujeres en la universidad data entre 1886 y $1889^{7} \mathrm{y}$, a pesar de que su acceso a estos espacios se presentó en una época temprana, no quiere decir que las mujeres accedían a los recintos universitarios de la misma manera y cantidad que en la educación normalista.

El 15 de febrero de 1971, Rosario Castellanos pronunciaba su discurso por la conmemoración del Día Internacional de la Mujer y, con él, una apertura abrumadora sobre la educación de las mujeres en México, y la desigualdad entre hombres y mujeres:

Los porcentajes comparativos de la instrucción elemental de hombres y mujeres no muestran muchas diferencias si se trata de grupos de campesinos, de artesanos y de obreros especializados. Pero si nos referimos a otras clases y nos elevamos a la enseñanza superior, las diferencias son algo más que apreciables: alarmantes. Un $85 \%$ de profesionistas varones contra un $16 \%$ de profesionistas mujeres. ${ }^{8}$

Lo que ella nos señalaba en 1971 eran dos cosas: primera, que la enseñanza superior estaba ocupada por "otras clases", quizá con mayor poder adquisitivo, un capital cultural más amplio o bien con mayores

6 Julia Tuñón. Mujeres. Entre la imagen y la acción (México: CONACULTA, 2015), 172.

7 Tuñón, Mujeres. Entre la imagen y la acción, 173.

8 Rosario Castellanos. "La abnegación: una virtud loca", Debate Feminista, Vol. 6 (1992): 287. 
posibilidades o privilegios sociales; $y$, segunda, que las mujeres ocupaban solo un pequeño porcentaje de dicha educación.

Así, la participación de las mujeres en la educación superior en México -llámense actualmente universidades federales, universidades estatales, normales públicas, centros de investigación, institutos tecnológicos, universidades politécnicas, universidades tecnológicas o universidades interculturales-, se fue presentando de manera diferenciada, ya que, por una parte, quedó supeditada al surgimiento de las instituciones y, por otra, a la normalización del género.

Blázquez nos muestra datos más precisos sobre los movimientos en la matrícula nacional en educación superior por género, de 1969 a 1985.

Para 1969 del total de alumnos, el $82.7 \%$ estaba constituido por hombres y el $17.3 \%$ por mujeres; para 1977 , la distribución porcentual pasó a $73.7 \%$ y $27.3 \%$ respectivamente; de 1977 a 1985 , la distribución fue de $65.5 \%$ y $34.5 \%$ respectivamente. ${ }^{9}$

Con ello, se puede observar de forma más copiosa la presencia de las mujeres en la educación superior en México entrada la década de 1980, ya que, con anterioridad, solo algunas cuántas lograban su ingreso a esos recintos.

El resto de las instituciones de educación superior, como el Instituto Politécnico Nacional, creado en 1936, como universidad tecnológica, mostró muy poca presencia de las mujeres, y su participación comenzó a ser más notoria en la década de 1990; esta ausencia aducía a que a las carreras de índole tecnológica se las consideraba socialmente más aptas para la población masculina.

$\mathrm{Y}$, a pesar de las crecientes investigaciones que se han realizado sobre el acceso de las mujeres a la universidad, ${ }^{10}$ aún quedan ausencias en la Historia de la educación de las mujeres; por ejemplo, las experiencias de

9 Norma Blázquez. "Incorporación de la mujer a la ciencia a comienzos de los noventa", en: La voluntad de ser. Mujeres en los noventa, comp. María Luisa Tárres. (México: El Colegio de México, 1997), 201.

${ }^{10}$ Patricia García. "La participación de la mujer en la educación: un camino escarpado". Revista GénEros, Vol. 9, № 26 (2002): 25-30; Patricia García. Mujeres académicas. El caso de una universidad estatal mexicana. (México: Plaza y Valdés, Universidad de Guadalajara, 2004); Sara Martínez. Mujeres y universidad. Vidas académicas (Colima: Universidad de Colima, 2006); Cristina Palomar, Feminizar no basta. Orden de género, equidad e inclusión en la educación superior (Ciudad de México: ANUIES, 2017)

Rev. His. Educ. Colomb-Vol. 25 № 25, julio-diciembre 2020-San Juan de Pasto-Colombia-ISSN 0123-7756-ISSN (Versión online) 2422-2348-pp. 117 - 145 
ingreso y egreso de quienes conformaron las primeras aulas de mujeres en las carreras universitarias, así como las profesoras formadoras dentro del mismo espacio.

En este sentido, la presencia de las mujeres como profesoras en las universidades se vio mermada por diversos factores: entre ellos, ver a la enseñanza superior como un espacio masculinizado a lo largo de la Historia y reconocido por sus sesgos en la diferenciación de género; es decir, considerar que las mujeres eran más aptas para trabajos relacionados con el cuidado de los otros, o por supuestos atributos "femeninos", como la paciencia y la mesura, pero no por el razonamiento o el pensamiento abstracto pretendido en los institutos científicos y literarios; a pesar de ello, podemos encontrar algunas mujeres que lograron su acceso a la universidad. En algunos estudios locales, se ubicó a algunas de ellas en tiempos posrevolucionarios, y cómo a varias las contrataron recién egresadas de su carrera universitaria. ${ }^{11}$

También, se debe advertir que la presencia de hombres y mujeres en el recinto universitario se contó en relación con la población de jóvenes en el Estado de San Luis Potosí. De acuerdo a las estadísticas históricas del Instituto Nacional de Estadística y Geografía, Censo de Población de 1960, se indica que se contaba con 131.310 hombres de 15 a 29 años de edad, y 137.326 mujeres en los mismos rangos de edad. ${ }^{12}$ Además, encontramos que la universidad contaba con una matrícula, para el ciclo escolar de 1964-1965, de 3690 estudiantes, de los cuales 1980 cursaban la Escuela Preparatoria adjunta a la institución; de esta cifra, no se tiene una desagregación por sexo. Sin embargo, cabe resaltar que, para la década de 1970, la universidad tuvo un crecimiento exponencial en su matrícula, que pasó, hacia 1975-1976, a 13.782 alumnos. ${ }^{13}$

Este acceso a la universidad representó, para las mujeres, una gran oportunidad en diferentes terrenos de su vida; tal como lo señalan Flecha y Palermo, y como lo veremos reflejado en las experiencias biográficas de nuestras entrevistadas:

${ }^{11}$ Blanca Susana Vega, "La participación de las estudiantes y profesoras en las aulas universitarias: San Luis Potosí, 1923-1952”, 113-136; Rosa María Huerta, "Ingreso y presencia de las mujeres en la matrícula universitaria en México", 282-306.

${ }^{12}$ Instituto Nacional de Estadística y Geografía, Censo de Población 1960. Consultado: https://www.inegi.org.mx/ programas/ccpv/1960/\#Tabulados

${ }^{13}$ Patrimonio y memoria. Universidad Autónoma de San Luis Potosí (San Luis Potosí: UASLP, 2016): 308 
Estudiar en la universidad tomó un doble sentido: desde el punto de vista del orden establecido, fue una puerta estrecha por la que solo unos pocos accedieron y tuvieron problemas. Pero desde el punto de vista de las mujeres fue una puerta amplia que aprovecharon, para formarse, ingresar a una profesión, y al pasar por la puerta, participar activamente en la vida social, política, económica y cultural de su tiempo. ${ }^{14}$

En este sentido, la participación educativa en la formación de otras y otros estudiantes les permitió, a su vez, posicionarse en sus actividades y devenires socio profesionales.

Este breve panorama sobre la educación superior universitaria de las mujeres nos lleva a reflexionar sobre las posibilidades que tuvieron algunas de las mujeres que accedieron a este nivel educativo, pero, también, a señalar las grandes diferencias que tuvieron con otros Sistemas Educativos superiores, como lo fue la educación superior normalista, en la cual las mujeres tuvieron mayor apertura y recepción para profesionalizarse en la enseñanza básica. Muestra de ello son los variados estudios que se tienen respecto a su educación y formación. ${ }^{15}$

Ahora bien, al presentar un estudio sobre educación de las mujeres y sus experiencias biográficas, es menester señalar la importancia que adquiere la memoria cuando se relata de forma oral, y cómo se da cuenta de las vivencias, rupturas y espacios expuestos en las narraciones de las entrevistadas que, además, transitan hacia su proceso de envejecimiento.

${ }^{14}$ Consuelo Flecha y Alicia Palermo, "Women at university. Strategies and achievements of a secular presence in Latin America and Spain", Culture \& History Digital Journal, 8, Vol. 1, e002. (2019): 1-15.

${ }^{15}$ Luz Elena Galván, Entre imaginarios y Utopias: historias de maestras (México: Publicaciones de la casa Chata, 2008); Debates y desafios de la historia de la educación en México (México, El Colegio Mexiquense, 2002); La educación superior de la mujer en México 1876-1940 (México, CIESAS, 1985); Oresta López, Que nuestras vidas hablen, Historias de vida de maestras y maestros indigenas tének y nahuas de San Luis Potosí (San Luis Potosí, COLSAN, 2010); Alfabeto y enseñanzas domésticas: el arte de ser maestra rural en el Valle del Mezquital (México, CIESAS-SEP-CONACYT, 2001); Adelina Arredondo, Obedecer, servir y resistir. La educación de las mujeres en la historia de México (México, Porrúa-UPN, 2003); María de Lourdes Alvarado, La educación superior femenina en el siglo XIX. Demanda social y reto gubernamental (México, CESU-UNAM, 2004); María del Carmen Gutiérrez, Abriendo brecha. Las mujeres en las escuelas profesionales del Estado de México (1917-1943) (Toluca, ISCEEM, 2010), entre otros. 


\section{La experiencia biográfica y la memoria}

En este apartado, es preciso señalar la indisociable relación entre la experiencia biográfica y la memoria, puesto que, en el campo de la Historia Oral, se privilegian las experiencias individuales y colectivas de los sujetos, que muchas de las veces no logran documentarse en los acervos y archivos ya existentes. Por ello, en la oralidad se conjuga la memoria, la historia individual, la historia colectiva y, con ellas, la identidad y la subjetividad a través de la palabra. Pero, ¿a qué nos referimos cuando hablamos de experiencia biográfica?

De acuerdo a Aceves, la experiencia biográfica se concibe como:

La organización de las vivencias en el transcurso del tiempo recordadas y narradas por la memoria del actor social, es decir, reconstruidas activamente desde el presente y referidas al pasado del individuo, ya sea en base a su propia autorreferencia, o bien, con base en su interrelación con las colectividades circundantes. La experiencia se refiere a lo recuperado y sistematizado por el individuo a lo largo de su existencia y en relación con el núcleo de vivencia recordado (el conjunto de relatos de vida que se vinculan en torno a un tema o motivo $[\ldots]) .^{16}$

Y no solo pensado desde la organización y sistematización de dichas experiencias, sino, también, respecto a lo que alude Arfuch:

[...] esa multiplicación de las voces donde lo vivencial, lo privado o lo íntimo se narran desde el registro de la "propia" experiencia, y adquieren así un innegable suplemento de valores: veracidad, autenticidad, proximidad, presencia. ${ }^{17}$

La memoria, ya lo señala Halbwachs, se vuelve reconstrucción, y la "reconstitución del pasado no puede jamás ser sino una aproximación;"

\footnotetext{
${ }^{16}$ Jorge Aceves. Usos de la historia oral y de vida en la investigación educativa. Aspectos metodológicos y fuentes orales (San Luis Potosí: El Colegio de San Luis, 2018), 27.

${ }^{17}$ Leonor Arfuch, "El espacio teórico de la narrativa: un desafío ético y político" Utopía y praxis latinoamericana. Revista internacional de filosofía iberoamericana y teoría social, Año 13, Núm. 42, (2008), 136.

${ }^{18}$ Maurice Halbwachs, Los marcos sociales de la memoria (Barcelona: Anthropos, 2004), 112.
} 
decir, la memoria solo es un acercamiento de lo acontecido, pero, al decirse o narrarse, se (re) construye.

En este sentido, podemos aducir que las experiencias biográficas y la memoria en mujeres mayores representa no solo configurar su identidad en el pasado, sino apropiarse en el presente de sus logros, rupturas y posiciones sociales respecto a su profesión y a su vida cotidiana; es decir, en la memoria y en la narración se reconstruye su identidad. Y esta identidad también se construye a partir de los otros, tal como la propia memoria, que es colectiva. Recordamos por y a través de los otros, como lo señala Mendoza, cuando cita a Ricoeur:

No recordamos solos, sino con ayuda de los recuerdos de los otros; los recuerdos propios se edifican sobre la base de los recuerdos de terceros. Y ocurre con cierta frecuencia que los recuerdos que uno considera propios en algún momento se han tomado de otros: "nuestros recuerdos se encuentran inscritos en relatos colectivos que, a su vez, son reforzados mediante conmemoraciones y celebraciones públicas de acontecimientos destacados". ${ }^{19}$

De ahí que las experiencias narradas por las profesoras se apoyen en recuerdos de otros, discursos de otros, palabras de otros, e incluso 'artefactos' que ayudan a rememorar, como lo fue la fotografía o, bien, documentos otros, tales como periódicos e incluso reseñas institucionales.

Así, también, la memoria y las experiencias biográficas no pueden ser separatistas, pero tampoco apolíticas; Iñigo lo relata:

Las construcciones de memoria tienen rendimientos para la configuración sociopolítica del presente. $\mathrm{O}$, en otras palabras, trabajan bajo el supuesto de que las elaboraciones del pasado están ancladas en el presente y pueden jugar roles tanto de resistencia y transformación del orden, como también de reproducción y perpetuación del mismo. ${ }^{20}$

${ }^{19}$ Jorge Mendoza, Sobre memoria colectiva. Marcos sociales, artefactos e historia (México: UPN, 2015), 20.

${ }^{20}$ Isadora Iñigo, "Sobre la relación entre memoria y política Contribuciones del pensamiento de Hannah Arendt para el campo de estudios de la memoria", Sophia Austral, No 22 (2018): 166. 
Con todo ello, es importante mencionar que las experiencias biográficas y la memoria de las mujeres mayores entrevistadas no solo merecen nombrarlas, sino, también, tienen un componente ético y político; es decir, las memorias también se reconocen como prácticas políticas, ya que "están orientadas por el desafío de generar un mundo común y de mantenerlo a través del tiempo". ${ }^{21}$

Esto lo veremos de manera más clara en el posicionamiento político de las profesoras entrevistadas, y que nos llevan a cuestionarnos sobre su memoria: ¿cómo se representan las experiencias en la memoria de mujeres en procesos de envejecimiento?, ¿cuál es el trabajo de la memoria en dichas experiencias? La función de la memoria en personas de edad avanzada puede reconocerse como una apuesta ética, pero, también, como un medio de restauración o instauración política de quien la rememora. Ética, porque conlleva una responsabilidad de quien escucha la evocación de los recuerdos, en el cuidado del otro y en el acercamiento a la intimidad de las personas. Y una política, porque la memoria de aquello recordado, pasado y presente, nos posiciona frente al otro, frente a lo colectivo, y configura, por lo tanto, nuestra identidad; es decir, la memoria de quien narra le permite posicionarse en un colectivo, para reconfigurar su identidad.

\section{Narrativas a través del tiempo. Perspectivas para pensar los relatos de las mujeres universitarias}

Delory-Momberger alude a que "lo narrativo es el lugar donde la existencia humana toma forma, donde se elabora y se experimenta bajo la forma de una historia." ${ }^{22}$ La narrativa, a través de la oralidad, es una forma asequible de construir la Historia de las mujeres. En este caso, las mujeres participantes fueron cuatro, cuyas características se enmarcan a continuación:

Tabla1. Profesoras entrevistadas.

\begin{tabular}{|c|c|c|c|c|c|}
\hline Nombre & Edad & Profesión & $\begin{array}{c}\text { Año de } \\
\text { ingreso a la } \\
\text { educación } \\
\text { superior }\end{array}$ & \multicolumn{1}{|c|}{ Campo laboral } & $\begin{array}{c}\text { Profesión de padre y } \\
\text { madre }\end{array}$ \\
\hline Hortensia & 85 años & Enfermera & 1952 & Profesora universitaria & $\begin{array}{l}\text { Padre: profesor nor- } \\
\text { malista } \\
\text { Madre: modista }\end{array}$ \\
\hline
\end{tabular}

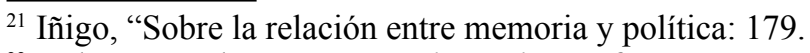

${ }^{22}$ Delory-Momberger, La condición biográfica, 5. 


\begin{tabular}{|l|l|l|c|l|l|}
\hline Mary & 84 años & $\begin{array}{l}\text { Ingeniera } \\
\text { química in- } \\
\text { dustrial }\end{array}$ & 1951 & $\begin{array}{l}\text { Profesora universitaria } \\
\text { trabajadora industrial }\end{array}$ & $\begin{array}{l}\text { Padre: médico } \\
\text { Madre: enfermera y } \\
\text { partera }\end{array}$ \\
\hline Luz María & 62 años & Abogada & 1972 & $\begin{array}{l}\text { Profesora universitaria } \\
\text { y trabajadora de go- } \\
\text { bierno }\end{array}$ & $\begin{array}{l}\text { Padre: empleado } \\
\text { empresa } \\
\text { Madre: profesora } \\
\text { normalista }\end{array}$ \\
\hline Amparo & 83 años & Médica & 1955 & $\begin{array}{l}\text { Profesora e investiga- } \\
\text { dora universitaria }\end{array}$ & $\begin{array}{l}\text { Padre: perito en } \\
\text { minería } \\
\text { Madre: sin } \\
\text { información precisa }\end{array}$ \\
\hline
\end{tabular}

Fuente: Esta investigación, con información de los relatos de vida.

Como un primer acercamiento a las profesoras, señalaremos algo de su origen familiar y social; posteriormente, nos narran cómo fue su ingreso a la educación superior y la elección de carrera; en un segundo apartado, a través de experiencias estudiantiles y laborales, nos enmarcan las dificultades en relación con su irrupción en espacios masculinizados, así como, también, sus posiciones frente a ello.

Con la finalidad de establecer una lectura conectada con las vivencias de las profesoras, los relatos se encuentran narrados por la investigadora y las palabras textuales de las entrevistadas anudadas a lo largo del texto.

\subsection{En la búsqueda del ingreso a la educación superior}

El ingreso de la educación superior universitaria de las entrevistadas se suscitó de formas particulares, pero resultó posible debido a diferentes factores: por ejemplo, las redes de apoyo, principalmente de los padres quienes contaban con un pensamiento mayormente liberal-, las condiciones sociales en las que ellas estaban inmersas y, por supuesto, la posición económica de la familia.

La enfermera y profesora universitaria Hortensia nació en la ciudad de Ríoverde, municipio de San Luis Potosí, en el año de 1935. Su padre fue profesor normalista y su madre modista y ama de casa. Tuvo cinco hermanos, tres hombres y dos mujeres; de ellos, solo tres fueron profesionistas: una maestra normalista, un médico y ella, como enfermera. Su padre, además de ser profesor normalista, se dedicó a la venta de medicamentos con la instalación de una farmacia; de ahí surgió su interés por la enfermería. 
En cuanto a la educación de las mujeres en su época y contexto social, la profesora comenta:

Bueno, ya en mi época ya iban muchas [mujeres] a la secundaria, pero ahí se quedaban; pocas eran, que tenían primaria y secundaria, sobre todo que en las ciudades o poblados [...] se iban [se matriculaban] a carreras de comercio o secretariado comercial, algo así, y profesoras de primaria. ${ }^{23}$

A pesar de la adversidad en la educación para las mujeres, la profesora realizó sus estudios de primaria y secundaria en su ciudad natal y, luego, viajó a la capital del Estado para estudiar la carrera de enfermería en la Universidad de San Luis. Ingresó a la carrera en la universidad en los años de 1952 a 1957. A su egreso de la universidad, la seleccionaron para ir a la ciudad de México a realizar una especialidad en docencia y que pudiera incorporarse a su término como instructora de enseñanza práctica de la carrera de enfermería, con incorporación en 1959 a la universidad. La Escuela Preparatoria la cursó en los años 1961 y 1962, después de terminar su carrera de enfermería y ya ser parte de la plantilla de la universidad. La decisión de estudiar la preparatoria cuando ya era una profesionista se debió a que ella, junto con otras enfermeras, plantearon ante el Consejo Universitario la posibilidad de que se implementara como requisito para cursar la carrera de enfermería la Preparatoria terminada, lo cual no se solicitaba con anterioridad, y ella misma no la presentaba.

Diez años después de ingresar a laborar en la universidad, la profesora solicitó un permiso para ausentarse durante ocho años y dedicarse a su familia. Pasado ese tiempo, se incorporó nuevamente a su trabajo como profesora de medio tiempo, lo cual le favorecía para dedicarse al cuidado de su familia.

$\mathrm{Su}$ interés por ingresar a la universidad y estudiar una carrera universitaria radicó principalmente por la motivación de su padre, quien deseaba que todos sus hijos tuvieran una carrera profesional.

Había muy pocas diferencias entre hombres y mujeres en mi familia, porque mi papá siempre tuvo en la mente que nosotras las mujeres estudiáramos [...] mi papá tenía la idea de que las mujeres, más sus hijas, sus hijos, fuéramos universitarios; no se le concedió que todos, pero mi papá decía que la mujer debe prepararse y debe tener una profesión en la que, soltera o casada, pueda trabajar.

${ }^{23}$ Entrevista a la enfermera Hortensia, San Luis Potosí, México, 18 de junio 2018. 
Por su parte, la ingeniera química Mary nació en San Luis Potosí, en el año de 1936; su padre fue de origen extranjero y médico de profesión, mientras que su madre de nacionalidad mexicana y con profesión de enfermera y partera.

La profesora cursó su educación primaria y secundaria en colegios privados y exclusivamente femeninos, en la capital potosina. Su educación preparatoria la realizó en los años de 1951 y 1952. Para ella y sus compañeras, el interés de ingresar a la universidad y pertenecer a una escuela mixta era notorio, así que cursaron la Escuela Preparatoria anexa a la universidad en el año de 1951; ella lo relató así:

[...] aunque ya había Preparatoria en el colegio, pues yo, igual que mis otras compañeras de secundaria, estábamos muy ilusionadas por entrar en la universidad. Ya teníamos en mente entrar a la universidad, y entramos a la Prepa bastantes compañeras [...] a nosotras nos hacía mucha ilusión tener compañeros; era uno de los atractivos, porque ahora afortunadamente ya la educación es mixta, pero anteriormente el colegio era solo para niñas. Y, ¿no?, pues nosotras ya queríamos ir a estudiar con los muchachos. Nos juntábamos para poder entrar a la universidad. ${ }^{24}$

La profesora Mary no optó por el área de la salud, como sus padres, pero sí eligió una profesión poco común para las mujeres de su época: ingeniera química industrial, por la cual optaban pocas mujeres, ya que no era una carrera feminizada, como había ocurrido con otras áreas de las Ciencias Químicas, como, por ejemplo, farmacología, en la cual su estudiantado era mayoritariamente femenino.

Era una Escuela, porque todavía no era Facultad, pero era una Escuela muy amigable, y como la mayoría de mis compañeras entraron por ese lado también, pues yo me sentía muy contenta, [...] en la carrera de Químicofarmacobiólogo había más mujeres; creo que mis compañeras no más tenían uno o dos compañeros hombres, ya en las Químicas Industriales predominaban los hombres y éramos pocas mujeres.

En lo tocante a la abogada Luz María, nació en San Luis Potosí en el año de 1956; su madre fue profesora normalista y su padre empleado

\footnotetext{
${ }^{24}$ Entrevista a la química industrial Mary, San Luis Potosí, México, 20 de junio 2018.
} 
en una empresa automotriz; tuvo 13 hermanos, 9 mujeres y 4 hombres. Toda su educación básica y media superior la estudió en el ámbito privado, excepto su educación superior, que cursó en la universidad pública.

A pesar de tener cuatro hermanos, en su vida escolar convivió en una casa en la que habitaban solo mujeres, ya que sus hermanos no vivían en la casa familiar.

Con ello, Luz María reconoció que, en su vida cotidiana y familiar, no hubo una distinción de género:

En la familia de mi papá, yo tuve una tía que nació en el nueve [1909] y estudió Ciencias Químicas en la universidad; entonces, si mi abuelo, que nació en el siglo diecinueve, se preocupó o quiso que su hija que nació en el nueve estudiara una carrera, pues era una familia que no limitaba mucho, mi tía aprendió a manejar, mi papá la enseñó a manejar un vehículo, patinaba, estudiaba inglés, ella era la única mujer en una familia con cuatro hombres, y era muy, muy cercana a nosotros; entonces, mi mamá también había estudiado una carrera, era maestra; entonces, la verdad, yo tuve un entorno muy privilegiado, y hasta muchos años después me di cuenta que no era común, que no todo mundo había nacido o había tenido un contexto tan poco restringido, o sea sin limitaciones por cuestiones de género; yo nunca supe que alguien no hiciera algo por ser mujer. ${ }^{25}$

La abogada Luz María señala que la elección de su carrera la hizo por "resignación", más no por "devoción;" decidió entrar a la carrera de derecho, en tanto pensaba qué estudios realizar; al fin, le agradó la carrera y decidió quedarse; la profesora no dio cuenta de que a esa carrera accedían pocas mujeres.

A decir de Huerta, la carrera de Derecho en la Universidad Autónoma de San Luis estuvo por mucho tiempo considerada propiamente masculina, y el ingreso y acceso de las mujeres a esa carrera fue de manera paulatina hasta ya entrada la década de 1970.

La Facultad de Derecho publicó una relación de egresadas de 1920 a 1975 (fecha en la que termina la lista). No fue hasta 1965 cuando hubo egresadas cada año. En los años setenta, se incrementó el número de egresadas al

${ }^{25}$ Entrevista a la abogada Luz María, San Luis Potosí, México, 13 de julio 2018. 
doble con respecto de los años anteriores. En 1974, terminaron sus estudios de Jurisprudencia 22 alumnas. $^{26}$

Este dato alude a nuestra entrevistada, ya que ella ingresó a la Escuela de Derecho en 1972, y formó parte de ese grupo minoritario de mujeres en dicha Escuela.

Por último, la doctora Amparo nació en Ciudad Victoria, Tamaulipas, en 1937. Allá realizó sus estudios primarios, secundarios y preparatorios. La doctora reconoció que la Preparatoria solo la cursaban pocas personas $\mathrm{y}$, en su generación, solo cuatro mujeres del total del grupo, dos de ellas a Ciencias Biológicas y dos alumnas más a Ciencias Químicas. Se mudó a la ciudad de San Luis Potosí e ingresó a la universidad en el año de 1955, pues contaba con el apoyo de sus padres para cursar su carrera. Fue la única universitaria de seis hermanas. Su elección de carrera la recordaba desde muy pequeña:

Le decía a mi mamá, cuando tenía 6 años: “¡Ay, yo quiero ser como el doctor, yo quiero ser doctor!". Siempre se supo en la casa que yo quería estudiar medicina. Es muy común que, cuando terminas la secundaria, te preguntan que con qué vas a seguir, pues allá, desde que entrabamos a la secundaria, empezábamos a decir qué era lo que íbamos a estudiar. ${ }^{27}$

El apoyo de sus padres, para la continuación de sus estudios, era claro y contundente:

Mi novio fue a hablar con mi mamá para pedirle permiso de que fuéramos novios; yo tenía 17 años, porque la Preparatoria era de dos años; en fin, cuando él fue, estuvo todo muy bien; a mi mamá le dio gusto que hubiera ido; le parecía un joven educado e hijo de doctor; entonces, él le dijo: "Nada más hay una cosa que quiero decirle: yo sé que ella se va a ir a estudiar a San Luis, pero yo quisiera que no estudiara medicina, que se quedara aquí y que me espere; ya, luego ,yo termino de estudiar y me vengo". Cuando dijo esto, mi mamá le contestó: "No, ella siempre ha querido estudiar medicina y hemos hecho todo lo posible para que ella estudie lo que quiere; ya, después de que ella termine, ya será cosa de ustedes, pero desde ahorita te digo que, con lo que dices, no estamos de acuerdo."

\footnotetext{
${ }^{26}$ Rosa María Huerta, "Ingreso y presencia de las mujeres en la matrícula universitaria en México", Revista de El Colegio de San Luis, No 14 (2017): 292.

${ }^{27}$ Entrevista a la doctora Amparo, San Luis Potosí, México, 20 de julio de 2018.
} 
En los relatos de vida de las cuatro profesoras universitarias, se debe notar que el apoyo tácito de la familia, la formación de sus padres, la posición económica y el pensamiento de lo que una mujer podía y debía ser, se tornaron fundamentales en su educación y posición frente a los otros.

\subsection{Irrumpir en un mundo escolar y laboral con mayor representación masculina}

La entrada al ámbito estudiantil y laboral que vivieron las cuatro profesoras se volvió complicada por las posibilidades y condiciones que vivieron. Si bien todas fueron profesoras universitarias, dos de ellas se dedicaron de tiempo completo a la universidad y las otras dos combinaban sus actividades de docencia con su profesión en otro espacio laboral.

En cada uno de los relatos de vida seleccionamos una experiencia sobre su vida cotidiana escolar y/o laboral en espacios masculinizados, los que las entrevistadas supieron sortear debido a la posición social y política que adquirieron al acceder a la educación superior.

La enfermera Hortensia narró, respecto a la relación médico-enfermera, maestro-alumna, algunas experiencias vividas en las relaciones de género que, con el paso del tiempo, le permitieron definir el espacio de acción entre la medicina y la enfermería; con ello, las enfermeras trataron de darle un posicionamiento distinto a su profesión.

Los maestros que estaban en ese momento, que eran médicos, nos miraban a nosotras lo que todo mundo sabemos; el médico, el jefe, y la enfermera, la ayudante del médico, el concepto antiguo que se tenía. Todavía existen ambientes de esa naturaleza.

Ya lo menciona Palomar, en relación con las mujeres y la ciencia; solo había dos caminos: el primero, era intentar acceder a la universidad, que era complicado; y el segundo, era ser una "invisible asistente" de algún hombre, ya fuera esposo, hermano o padre; ${ }^{28}$ en este contexto, diríamos médico o maestro.

${ }^{28}$ Cristina Palomar, "Maternidad y mundo académico", Alteridades, Vol. 19, No 38 (2009): 61. 
Los médicos maestros nos decían: "Ustedes, si saben, ustedes son producto de nosotros" [...] en general, la relación médico-enfermera fue siempre muy buena, no hubo roces ni nada, siempre respetando; los que a veces se ponían un poquito difíciles eran los residentes o los internos, a veces se portaban muy superiores. Pero, también, las enfermeras tienen que darse su lugar. Una compañera le dijo a un médico: "A mí no me grite”, porque sí hay casos en los que el médico se siente muy superior a la enfermera y le dice: "Tú, obedece"; se ven a veces como el jerarca.

La resistencia a quedar posicionadas en este papel motivó a las enfermeras -entre ellas, la enfermera Hortensia- a asumir su profesión y un cambio de pensamiento de otra manera. Con el paso del tiempo, dentro de la universidad se dejó de llamar a las enfermeras como "instructoras o señoritas" y se dio paso a llamarlas "maestras".

En la carrera de enfermería, la lucha por posicionar a la disciplina dio inicio a una nueva forma de ver a la enfermería, ahora como una profesión y no como una ayudantía médica.

A la carrera de enfermería -como muchas otras en la universidad- la dirigían profesores varones en las clases teóricas; sin embargo, las clases prácticas de hospital las impartían enfermeras titulares. A pesar de ello, algunas profesoras estaban incorporadas a la Escuela de Medicina y colaboraban, también, en la carrera de enfermería e impartían las clases teóricas. Con ellas, la profesora Hortensia pudo establecer lazos importantes que le permitieron continuar su carrera, a pesar de las dificultades.

Por su parte, la profesora Mary trató de pertenecer a la industria; sin embargo, era consciente de que, en su tiempo, no se empleaban mujeres en las empresas químicas:

Cuando yo terminé, hice mis primeras prácticas en la Fundición de Morales, en la planta de cobre; era rarísimo que uno pudiera hacer prácticas ahí, pero mi papá había tenido relaciones con los jefes y con el gerente [...] en una ocasión, una de las pruebas piloto, me brincó una resina a la cara y me quemé, cualquier cosilla, pero no tenía lentes de seguridad y dije: "No, aquí se acaba," y mi papá me mandó a Europa por un tiempo. Entonces, yo estuve como seis meses y viajé y me gustó muchísimo; practiqué el francés e inglés y, cuando llegué, yo quería conseguir trabajo, pero en ninguna industria de San Luis querían emplear mujeres. 
La industria era poca en la capital de San Luis Potosí para esa época; además del ferrocarril, tenía fuerte presencia la ASARCO (American Smelting and Refining Company), ambas consideradas buenas fuentes de empleo.

Al egresar de la universidad hacia 1957, las egresadas buscaban posibilidades laborales y, aunque la profesora Mary tenía interés en la investigación, las oportunidades en ese campo eran muy limitadas. Además, las desigualdades laborales la orillaron a buscar otras opciones de trabajo alejadas de su profesión.

Me hablaron de la ASARCO para decirme que si me quería hacer cargo de la escuelita americana; fue entonces cuando fui maestra de primaria, que realmente fui institutriz [...] tenía niños desde primero hasta séptimo, lo que cayera, porque esta escuelita fue preparada para los hijos de los ingenieros que estaban trabajando para la compañía; para que no se fueran a Estados Unidos por razón de la educación de los niños, ahí se les daba la educación.

Sin embargo, años después le ofrecieron, dentro de misma industria, el trabajo de química industrial, que aceptó y la llevó a convertirse en una de las primeras mujeres trabajadoras en espacios empleados solo por el sexo masculino.

[...] la escuelita se cerró, y el gerente me preguntó: -Tú eres química, ¿te gustaría trabajar en el Laboratorio? Pasarías con tu mismo sueldo de la escuelita americana y, cuando se desocupe alguien, entonces tú serás ayudante de químico. Yo le dije que sí. Fue un cambio del cielo a la tierra, porque yo era la mera jefa y así empecé a trabajar y en un mundo de hombres absolutamente; entonces, así fue como yo entré al ASARCO y fui la primera mujer que trabajó en la ASARCO, fuera de las enfermeras o de las maestras o de las cocineras, porque no había ni secretarias, no se usaba; los señores eran lo que le llaman jefe de oficina. En fin, ya poco a poco, después de unos dos o tres años, no sé cuánto realmente, se recibió una amiga mía de ingeniera química y ya realmente a ella ya la contrataron como ingeniera química y ya, entonces, nos pusieron un baño; no había baño para mujeres y, pues, yo tenía mi llavecita de la escuela y tenía que travesar un campo para ir al baño. Es lo que le pasa a uno; también, cuando me mandaron a Peñoles [otra industria], en la planta no había baños para mujeres. 
Como señalan Ibarra y Castellanos ${ }^{29}$ las mujeres se enfrentaron a las creencias de la sociedad, para romper con el estereotipo de que ciertas ocupaciones son exclusivas para el género masculino, a pesar de seguir con las bases de un sistema cultural que diferencia las potencialidades masculinas de las femeninas, lo que genera que las mujeres no opten por las ciencias exactas.

Su conexión con la universidad confluyó algunos años después, aunque no como profesora, por su formación, sino como profesora de inglés.

Yo empecé a dar clases, también, [en la UASLP] y mi primera clase fue inglés técnico; o sea, que estábamos ahí en la ASARCO hasta las tres de la tarde y a las cinco ya estamos en la escuela [Escuela de Ciencias Químicas], casi todos los mismos; fue bonito ese intercambio.

Con la abogada Luz María, las cosas no fueron muy diferentes; ella comienza la búsqueda de trabajo, que no le fue complicado, ya que el primer día que salió en su búsqueda consiguió su primer nombramiento, Ministerio Público, en un municipio del Estado de San Luis Potosí. De ahí en adelante, la abogada ejerció su carrera de manera ininterrumpida; como ella lo señala:

Del 79 [1979] al 2012, digamos que, de manera ininterrumpida, he trabajado, salvo dos semanas en octubre del 99 [1999], en lo que me fui a la Procuraduría [...] y en lo que salgo de ahí para irme a la Defensoría pasaron cuatro días; entonces, salvo dos semanas y cuatro días, todo mi trabajo ha sido de corrido; hasta ahora, llevo 39 años de servicio.

Además de su práctica en su ámbito profesional, la abogada ejerció la práctica docente en la Facultad de Derecho de la UASLP, espacio reservado también para el sexo masculino, en el cual, dicho sea de paso, sigue siendo mayormente ocupado por hombres; de 247 docentes en la Facultad de Derecho de la UASLP, solo 63 son profesoras. ${ }^{30}$

\footnotetext{
${ }^{29}$ Eugenia Ibarra y Gabriela Castellanos "Género y educación superior. Un análisis de la participación de las mujeres como profesoras en la Universidad del Valle", Revista La manzana de la discordia, Vol. 4, No 1 (2009): 81.

${ }^{30}$ Informe Rectoría, 2018-2019. Universidad Autónoma de San Luis Potosí. http://www. uaslp.mx/Informes/ Documents/2018-2019-06-Indicadores.pdf
} 
Hasta ahora, mi Facultad no ha tenido ni una presidenta de Sociedad de Alumnos, mujer; no ha tenido ni una Consejera alumna, no ha tenido ni una presidenta de la Asociación de Personal Académico; ahora, tenemos a la primera Consejera mujer [...] Desde los 90's, en la Escuela hay más alumnas mujeres que hombres; y en la planta docente, ahora sí hay más mujeres, pero teníamos una planta de 114 o 120 y no llegaban a 18 mujeres, cuando ya las alumnas iban arriba del 50\%; entonces, yo sí creo que la universidad ha sido más difícil para la mujeres [en la docencia].

Una experiencia más que comparte la abogada es la violencia ejercida, a modo de broma, que le jugaron sus compañeros de trabajo, cuando ocupaba ya el cargo de Ministerio Público.

Llego al Ministerio Público el primer día; me dicen: "Ni modo, ya te tocó; tienes que ir a dar fe de un cadáver al hospital central"; en ese tiempo no había ambulancias de Procuraduría, te llevaban los de las funerarias; entonces, cuando llegamos al anfiteatro, bajamos a donde están las gavetas, los refrigeradores; de manera muy cortés, me dicen: "Pásale”, pero, a la hora que entro, se cierra la puerta; entonces, estaban los refrigeradores cerrados y yo sabía que tenían cuerpos; me siento en el piso, me recargo en la puerta cerrada y empiezo a rezar; cierro los ojos y me digo: "No les voy a gritar ni les voy a tocar desesperada ni nada". Se apaga la luz, y no me ganaron; hubo un momento en el que me dijeron: “¡Ay!, Licenciada, ¡creo que se cerró la puerta!". Me doy el patín y dije que no me había dado cuenta, pues yo estaba esperando [...] En realidad, ahora puedo tener información y puedo ver muchas cosas que no vi, que pasé sin ver, que ni en mi condición de madre soltera vi; yo no digo que no haya habido [la discriminación]; no vi, no sentí, porque no estaba educada a sentir ni a ver.

Por su parte, la profesora Amparo recuerda las dificultades de estar en una Escuela masculinizada, como lo fue Medicina, además de los abusos ejercidos por los profesores hacia las alumnas, y los métodos de evaluación a base de infundir miedo o intimidación; eran las razones por las que algunas mujeres desistían de continuar con su carrera.

El ¿por qué las mujeres íbamos disminuyendo en el grupo? Porque algunos profesores regañaban tan feo a las mujeres, que algunas se salían; te estoy diciendo esto porque yo viví que un profesor le dijo a una amiga: "Mientras yo sea aquí el profesor, usted no pasará" y mi amiga se salió; ella era una 
enfermera que quería estudiar medicina, era muy dedicada; a mí me dio mucho coraje esa vez [...] Después, supe algo sobre otra compañera. Un doctor, creo que fue el mismo, ella no podía comprar el libro que se necesitaba en la clase, y el doctor le dijo: "Yo tengo una copia en mi consultorio; puede pasar por ella, por favor". Resulta que se quería aprovechar; entonces, mi compañera dijo que no; se dio cuenta de quién era el doctor. Bueno, ya estoy mezclando muchas cosas, pero, en esa época, se notaba cuando un profesor quería aprovecharse de las circunstancias [...] Las dificultades que las mujeres tuvimos son las mismas de hoy, nada más que hoy, como que esto sí se da a conocer; antes era algo que se mantenía entre plática solamente, pero no había un reporte, nadie se atrevía a reportar; esa fue la razón por la que compañeras se iban retirando; de por sí que éramos poquitas y ya, al final, en total quedamos solo dos.

Las narrativas de las profesoras nos muestran que su irrupción en los espacios tradicionalmente masculinos devinieron en diferentes, sutiles y continuas formas de violentarlas: desde subestimar su profesión, ser solo una ayuda, no ejercer por ser mujer, no poder ocupar cargos en la toma de decisiones; transgredir, aduciendo a bromas o chistes por ser mujer, o bien, acosar psicológica y sexualmente, fueron algunas de las violencias que vivieron las profesoras en los espacios escolares y laborales, lugares no pensados para su presencia, y mucho menos para aportar conocimiento.

Lo que encontramos en estos relatos son experiencias de estar en espacios en los que constantemente había que permanecer de pie y romper con los estereotipos establecidos; las posturas de las profesoras fueron de resistencia y lucha, pero, también, de un reconocimiento en sus privilegios, de su origen y de sus condiciones sociales.

En este sentido, podemos vincular que la educación que las profesoras entrevistadas tuvieron respecto a otras de su tiempo y contexto local fueron mayormente predilectas, y que la memoria y las experiencias biográficas que narran en su proceso de envejecimiento reconstruyen su identidad y su posición política en lo colectivo y en lo gremial.

\section{Conclusiones}

Para nuestras entrevistadas, el acceso a la educación secundaria, preparatoria, y superior universitaria, más sus condiciones de vida, deja a la luz su situación privilegiada en relación con el contexto social e histórico 
de las mujeres. Y, a pesar de ello, se logran ver en sus narrativas algunas circunstancias poco favorecedoras, que se sobrellevaron.

A través de la experiencia biográfica, advertimos que pertenecieron a un grupo social que creó posibilidades para otras mujeres y que, al identificar las realidades que ellas vivieron, también podemos entender las realidades actuales de las mujeres.

Bolufer nos señala cómo el enfoque biográfico es indispensable para pensar en las mujeres como aquellas que puede establecer rupturas con el orden de género:

El enfoque biográfico resulta especialmente pertinente en historia de las mujeres, en la medida en que permite presentar a estas no como víctimas pasivas de un orden desigual, sino como sujetos activos en el seno del mismo, y matizar el peso de las normas y convenciones - entre ellas las de género- con frecuencia presentadas como marcos fijos y determinantes.

Las variantes de género, educación y trabajo se entrecruzan para mostrarnos lo que implicaba ser mujer y aspirar a ser profesionista y, aún con dificultades, ejercer su profesión.

Las cuatro narrativas nos muestran circunstancias similares, que les permitieron acceder a una educación superior universitaria: provenir de padres profesionistas, con una estabilidad económica, tener acceso a una instrucción privada en los primeros años de su vida escolar, venir de una familia con un pensamiento mayormente liberal y/o la oportunidad de dominar otra lengua. Ello nos lleva a reflexionar en la cadena de acontecimientos/privilegios que les permitió relacionarse y posicionarse; incluso lo apunta la abogada Luz María:

Yo nunca vi que hubiera límites; yo decía: "Si yo lo he hecho, lo puede hacer cualquiera", y me tardé muchos años en darme cuenta que no, ya que muchas nacen en una casa donde les dicen que por ser mujer no, y que ese no había sido mi contexto, pero me tardé muchísimos años. [...] En la Procuraduría de delitos sexuales y violencia familiar es cuando yo descubro y aprendo muchas cosas, no de Derecho sino de Humanidad, y donde me di cuenta, donde valoré el privilegio que yo había tenido. [...] Yo no soy una heroína por hacer lo que hago o por estar en donde estoy, porque ya venía cargada con todo el equipamiento necesario; 
entonces, la verdad, a mí me costó mucho trabajo y muchos años darme cuenta de que realmente vivimos en un mundo inequitativo. [...]

Las reflexiones de la abogada Luz María nos ayudan a reconocer que el acceso de las mujeres a la educación superior universitaria fue, como lo repara García, "un logro de una selecta minoría, que se encontraba en circunstancias muy específicas".

De acuerdo a los relatos presentados, podemos dar cuenta que el acceso de las mujeres a la educación superior en los años ya descritos estuvo envuelto en dificultades, pero, también, de osadías; es decir, de experiencias que cuentan cómo las profesoras se atrevieron a incursionar en espacios que aún no eran bien vistos para las mujeres.

Registrar las historias de estas mujeres es reconocer que abrieron camino para la educación de las mujeres que les sucedieron. A su vez, también nos muestra cómo ellas formaron parte de la transformación y apertura de nuevas oportunidades de estudio en espacios que hasta entonces los habían controlado y ocupado los hombres.

Por último, se debe señalar que la posición política de las mujeres, al narrar sus experiencias, se ve reflejada en lo que Flecha y Palermo aluden:

El tiempo ha demostrado que esta educación ayudó a innumerables mujeres no solo intelectual y profesionalmente, sino también a tomar conciencia de cómo se programaba su vida desde fuera. Esto fortaleció su voluntad de introducir cambios en las órdenes que recibían.

Es decir, al escuchar las voces de las profesoras y sus experiencias, damos cuenta de su posición política, de su construcción y reconstrucción al verse inmersas dentro de un gremio, de un colectivo minoritario, en el que aún se busca que las reconocieran y escucharan. En el que aún se sigue luchando por una mayor apertura y una disolución de los círculos predilectos en la educación superior.

\section{Referencias}

\section{Fuentes orales}

Entrevista a Hortensia, San Luis Potosí, México, 18 de junio de 2018.

Entrevista a Mary, San Luis Potosí, México, 20 junio de 2018. 
Entrevista a Luz María, San Luis Potosí, México, 13 de julio de 2018.

Entrevista a Amparo, San Luis Potosí, México, 20 de julio de 2018.

\section{Bibliografía}

Alvarado, María de Lourdes. La educación superior femenina en el siglo XIX. Demanda social y reto gubernamental. México: CESU-UNAM, 2004.

Arfuch, Leonor. "El espacio teórico de la narrativa: un desafío ético y político". Utopía y praxis latinoamericana. Revista internacional de filosofia iberoamericana y teoría social, Año 13, No 42, (2008): 131-140.

Arredondo, María Adelina. Obedecer, servir y resistir. La educación de las mujeres en la historia de México. México: Porrúa-UPN, 2003.

Blázquez, Norma. "Incorporación de la mujer a la ciencia a comienzos de los noventa". En: La voluntad de ser. Mujeres en los noventa, compilado por Tarrés, María Luisa. México: El Colegio de México, 1997. 195-210.

Bolufer, Mónica "Multitudes del yo: biografía e historia de las mujeres". Ayer, Vol. 93, N 1, (2014): 85-116.

Calveiro, Pilar. "Los usos políticos de la memoria". En: Sujetos sociales y nuevas formas de protesta en la historia reciente de América Latina, compilado por Caetano, Gerardo. Buenos Aires: CLACSO, 2006. 359-386.

Castellanos, Rosario. "La abnegación: una virtud loca”. Debate Feminista, Vol. 6, (1992): 287-292.

Delory-Momberger, Christine. La condición biográfica. Medellín: Universidad de Antioquia, 2015.

Flecha, Consuelo y Palermo, Alicia. "Women at university. Strategies and achievements of a secular presence in Latin America and Spain". Culture \& History Digital Journal, Vol. 8, No 1, (2019): 1-15.

Galván, Luz Elena y López, Oresta. Entre imaginarios y Utopias: historias de maestras. México: Publicaciones de la casa Chata, 2008. 
Galván, Luz Elena. Debates y desafios de la historia de la educación en México. México: El Colegio Mexiquense, 2002.

Galván, Luz Elena. La educación de la mujer en México, 1876-1940. México: CIESAS, 1985.

García, Patricia. "La participación de la mujer en la educación: un camino escarpado". Revista GénEros, Vol. 9, № 26, (2002): 25-30.

García, Patricia. Mujeres académicas. El caso de una universidad estatal mexicana. México: Plaza y Valdés- Universidad de Guadalajara, 2004.

Gutiérrez, María del Carmen. Abriendo brecha. Las mujeres en las escuelas profesionales del Estado de México (1917-1943). Toluca: ISCEEM, 2010.

Halbwachs, Maurice. Los marcos sociales de la memoria. Barcelona: Anthropos, 2004.

Huerta, Rosa María. "Ingreso y presencia de las mujeres en la matrícula universitaria en México". Revista de El Colegio de San Luis, No 14, (2017): 282-306.

Ibarra, Eugenia y Castellanos, Gabriela. "Género y educación superior. Un análisis de la participación de las mujeres como profesoras en la Universidad del Valle”. Revista La Manzana de la Discordia, Vol. 4, No 1, (2009): 73-92.

Informe de rectoría 2018-2019. Universidad Autónoma de San Luis Potosí. http:// www.uaslp.mx/Informes/ Documents/2018-2019-06-Indicadores.pdf

Instituto Nacional de Estadística y Geografía, Censo de Población 1960. https:// www.inegi.org.mx/programas/ccpv/ 1960/\#Tabulados

Iñigo, Isidora. "Sobre la relación entre memoria y política Contribuciones del pensamiento de Hannah Arendt para el campo de estudios de la memoria". Sophia Austral, № 22, (2018): 163-181.

López, Oresta, Que nuestras vidas hablen, Historias de vida de maestras y maestros indigenas tének y nahuas de San Luis Potosí. San Luis Potosí: COLSAN, 2010.

López, Oresta, Alfabeto y enseñanzas domésticas: el arte de ser maestra rural en el Valle del Mezquital. México: CIESAS-SEP-CONACYT, 2001. 
Martínez, Sara. Mujeres y universidad. Vidas académicas. Colima: Universidad de Colima, 2006.

Mendoza, Jorge. Sobre memoria colectiva. Marcos sociales, artefactos e historia. México: UPN, 2015.

Palomar, Cristina. "Maternidad y mundo académico". Alteridades, Vol. 19, N 38, (2009): 55-73.

Palomar, Cristina. Feminizar no basta. Orden de género, equidad e inclusión en la educación superior. México: ANUIES, 2017.

Patrimonio y memoria. San Luis Potosí: Universidad Autónoma de San Luis Potosí, 2016.

Tuñón, Julia. Mujeres. Entre la imagen y la acción. México: CONACULTA, 2015.

Vega, Blanca Susana, "La participación de las estudiantes y profesoras en las aulas universitarias: San Luis Potosí, 1923-1952” En: Los mundos escolares en el noreste de México: ruralidad y género en el siglo XX. Coordinado por Chávez, Mónica y Medina, René. México: UNAM-BECENE, 2019. 113136.

\section{Citar este artículo}

Vega, Blanca Susana. "Mujeres en la universidad: presencia y experiencias biográficas San Luis Potosí, México 1950-1970”. Revista Historia de la Educación Colombiana. Vol. 25 No 25 (2020): 117-145. DOI: https://doi. org/10.22267/rhec.202525.81 